\title{
Correlation study of DNA methylation of WNT6 gene with osteosarcoma in children
}

\author{
$\mathrm{LI} \mathrm{LI}^{1 *}, \mathrm{CHI} \mathrm{XU}^{2 *}$, PINGTAO LIU ${ }^{1}$ and JIA HUANG ${ }^{3}$ \\ ${ }^{1}$ Department I of Orthopedics, Jingmen No. 2 People's Hospital, Jingmen, Hubei 448000; ${ }^{2}$ School of Health Sciences, \\ Wuhan University, Wuhan, Hubei 430000; ${ }^{3}$ Department IV of Orthopedics, Jingmen No. 2 People's Hospital, \\ Jingmen, Hubei 448000, P.R. China
}

Received December 20,2016; Accepted April 11, 2017

DOI: $10.3892 / \mathrm{ol} .2017 .6135$

\begin{abstract}
The aim of this study was to evaluate the status and explore the impact of DNA methylation of WNT6 gene with osteosarcoma in children. A total of 50 patients with primary osteosarcoma in children were enrolled. The expression of WNT6 protein was determined by immunohistochemical staining. The DNA methylation level of WNT6 gene was evaluated by methylation-specific PCR (MSP). Human MG63 osteosarcoma cells and human normal Hfob1.19 osteoblasts were selected and cultured. Western blot analysis was utilized to measure the expression of WNT6 in the two cell lines. MSP was used to assess the status of DNA methylation of WNT6 gene. The correlation between DNA methylation of WNT6 gene and prognosis of osteosarcoma in children was evaluated by statistical analysis of the clinical and prognostic data. The results of the immunohistochemical assay showed that $84 \%(42 / 50)$ of primary osteosarcoma was WNT6 positive. MSP indicated that DNA methylation of WNT6 gene was found in 9 cases $(18 \%)$ of primary osteosarcoma. Western blot analysis showed that WNT6 protein expression in MG63 cells was significantly higher than that in normal human Hfob1.19 osteoblasts, whereas, the level of DNA methylation of WNT6 gene in MG63 cells was significantly lower than in Hfob1.19 cells. Analysis of survival, prognosis and their correlation found that the DNA methylation level of WNT6 gene was
\end{abstract}

Correspondence to: Dr Pingtao Liu, Department I of Orthopedics, Jingmen No. 2 People's Hospital, 39 Xiangshan Road, Jingmen, Hubei 448000, P.R. China

E-mail: pvxjlr@163.com

Dr Jia Huang, Department IV of Orthopedics, Jingmen No. 2 People's Hospital, 39 Xiangshan Road, Jingmen, Hubei 448000, P.R. China

E-mail: vvbljb@163.com

*Contributed equally

Key words: WNT6, osteosarcoma, MG63 cell line, methylation, gene alteration negatively correlated with the prognosis of children with osteosarcoma. In conclusion, there was a high level of WNT6 gene expression in primary osteosarcoma, which was mainly due to low DNA methylation level of WNT6 gene. The DNA methylation of WNT6 gene was negatively correlated with the prognosis of patients with osteosarcoma in children.

\section{Introduction}

Osteosarcoma is a common primary parenchymal bone tumor that occurs mainly in adolescents, of which incidence in male is slightly higher than that in female (1-3). Osteosarcoma has characteristics of local infiltration and high incidence of early metastasis with $\sim 80 \%$ of patients experiencing pulmonary metastasis (4). The incidence of osteosarcoma in children and adolescents is ranked sixth, but the mortality caused by osteosarcoma in children and adolescents is ranked second (5). With the development of medical sciences, the 5-year survival rate of osteosarcoma patients can reach $60 \%$, but the 5 -year event-free survival accounts for only $20 \%$ (6).

The WNT signaling pathway involves a number of proteins, through which the network is very complex and plays a key role in cell proliferation, growth and differentiation (7). Alteration of related genes in the WNT signaling pathway can affect transcription and expression of the target gene downstream, where gene mutation is involved in the development of osteosarcoma (8). The WNT6 gene is a member of the WNT gene family that can encode a polypeptide protein with 365 amino acids. WNT6 is involved in various physiological or pathological processes through the WNT- $\beta$-catenin-TCF signaling pathway (9-11). WNT6 was found to relate with the development of teeth, muscles and cardiac muscles, and differentiation of human corneal stem cells and embryonic stem cells (12-16). WNT6 gene expression in colorectal adenoma was significantly increased, which was positively correlated with degrees of tumor malignancy (17).

Extensive studies confirmed that the abnormal expression of related genes in the WNT signaling pathway is associated with DNA methylation of the gene. It may be hypothesized that the difference of WNT6 expression in normal and tumor tissues may be related to DNA methylation of the gene. In order to verify this inference, the correlation between DNA methylation of WNT6 gene and osteosarcoma in children was studied. 
The expression of WNT6 protein in primary osteosarcoma in children and the DNA methylation level of WNT6 gene were evaluated by immunohistochemical staining and methylationspecific PCR (MSP), respectively. In addition, the expression of WNT6 protein and the DNA methylation of WNT6 gene in MG63 and Hfob1.19 cells were analyzed by western blot analysis and MSP, respectively. The follow-up data was statistically analyzed to assess the correlation between DNA methylation of WNT6 gene and prognosis of osteosarcoma in children, which provided a basis for DNA methylation of WNT6 gene serving as a diagnostic marker for osteosarcoma in children and predicting prognosis.

\section{Materials and methods}

Materials. The selected cells and materials were obtained from commercial sources. The human MG63 osteosarcoma cells and human normal Hfob1.19 osteoblasts were obtained from the Chinese Academy of Sciences Cell Bank, Beijing, China; DMEM culture medium was obtained from Invitrogen Life Technologies, Carlsbad, CA, USA; fetal bovine serum was obtained from Hyclone, Logan, UT, USA; PCR chIP kit for WNT signaling pathway DNA methylation was obtained from SABiosciences, Los Angeles, CA, USA; DNA extraction kit was obtained from Qiagen, Dusseldorf, Nordrhein-Westfalen, Germany; WNT6, GAPDH primary antibody, HRP-labeled secondary antibody, and protein extraction kit were obtained from ProteinTech Group, Inc., Wuhan, China; primers were obtained from Takara Bio (Dalian) Co., Ltd., Dalian, China; immunohistochemical staining kit SP-9001 were obtained from Beijing Zhongsheng Jinqiao Biotechnology Co., Ltd., Beijing, China.

Archived tumor tissue specimens of patients with primary osteosarcoma who were admitted to the Department of Orthopedics of Jingmen No. 2 People's Hospital from January 2008 to December 2010 were chosen for clinical study, including paraffin-embedded specimens and fresh tumor specimens preserved in liquid nitrogen. A total of 50 patients with age range from 8 to 14 years were enrolled, including 27 males and 23 females, aged 8-14 years, who were clinically and pathologically diagnosed with primary osteosarcoma and treated by surgery for the first time without chemotherapy. Parents of all involved patients signed informed consent. The study was approved by the Ethics Committee of Jingmen No. 2 People's Hospital.

Cell culture. MG63 and Hfob1.19 cells were cultured in DMEM containing $10 \%$ fetal bovine serum at $37^{\circ} \mathrm{C}$ with $5 \%$ $\mathrm{CO}_{2}$. The medium was changed every $24 \mathrm{~h}$. The cells were passaged by trypsin treatment at $80 \%$ confluency.

Immunohistochemical measurement of WNT6 protein expression in pathological tissues. Following the instructions of the immunohistochemical staining kit, paraffin sections containing tumor tissue were dewaxed and treated with $3 \%$ $\mathrm{H}_{2} \mathrm{O}_{2}$ to inactivate the endogenous peroxidase. The obtained tissue sections were antigen retrieval, serum blocking and mixed with rabbit polyclonal WNT6 antibody (dilution, 1:100; cat. no. ab150588; Abcam, Cambridge, MA, USA). Subsequently, the above tissue sections were incubated
Table I. Primer sequences for DNA methylation and unmethylation of WNT6 gene.

\begin{tabular}{llll}
\hline Primers & & Sequences & $\begin{array}{c}\text { Size } \\
\text { (bp) }\end{array}$ \\
\hline WNT6-M & F & 5'-GTTGTCGTTTTTATTTTTTCGTTTC-3' & 195 \\
& R & 5'-ACTAACTCTACCACCACTTCCGAC-3' & \\
WNT6-U & F & 5'-TGTTGTTTTTATTTTTTTGTTTTGG-3' & 194 \\
& R & 5'-AACTAACTCTACCACCACTTCCAAC-3' & \\
\hline
\end{tabular}

overnight at $4^{\circ} \mathrm{C}$, rinsed with PBS three times, and mixed with the secondary goat anti-rabbit (HRP) IgG antibody (dilution, 1:2,000; cat. no. ab6721; Abcam). After incubation for $30 \mathrm{~min}$ the tissue sections were rinsed three times with PBS, DAB staining, slide mounting with balsam, and photographing under a microscope (TE2000-U; Nikon Corp., Tokyo, Japan).

WNT6 protein staining was positive if brown particles were seen on the cell membrane or the cytoplasm. Scoring was according to the following criteria: negative (-) if no or $<5 \%$ positive cell staining, weakly positive $(+)$ if $\leq 50 \%$ positive cell staining, strongly positive $(++)$ if $\geq 51 \%$ positive cell staining. The results were analyzed statistically.

MSP measurement of DNA methylation of WNT6 gene in pathological tissues. Patient tissue specimens were selected. Total DNA was extracted using the DNA extraction kit. The purity and concentration of the extracted DNA were determined by ultraviolet spectrophotometry (U-3010; Hitachi Co., Tokyo, Japan). Primer design and synthesis were completed by Takara Bio (Dalian) Co., Ltd. The primer sequences are shown in Table I. WNT signaling pathway DNA methylation PCR kit was used for measurement of DNA methylation of WNT6 gene. The procedure included restriction endonuclease digestion and real-time quantitative PCR. PCR conditions were as follows: $95^{\circ} \mathrm{C}$ for $5 \mathrm{~min}$; then 35 cycles of $95^{\circ} \mathrm{C}$ for $15 \mathrm{sec} ; 60^{\circ} \mathrm{C}$ for $35 \mathrm{sec}$; and finally $72^{\circ} \mathrm{C}$ for $5 \mathrm{~min}$. The results were processed statistically following the data processing method in the manual.

Western blot measurement of WNT6 protein expression in cells. The cultured MG63 and Hfob1.19 cells were broken open by adding cell lysate. The total protein was extracted according to the instructions of the protein extraction kit, and the protein concentration was measured. The proteins of the sample were separated using SDS-PAGE and transferred to the membrane using a wet system. WNT6 and GAPDH (1:500 diluted) antibodies were added, respectively, after the membrane was blocked with $5 \%$ BSA. The membrane was incubated overnight at $4^{\circ} \mathrm{C}$ followed by the addition of the HRP-labeled secondary antibody and incubation for $2 \mathrm{~h}$. The ECL developer was applied to the membrane in a dark chamber for $1 \mathrm{~min}$. A gel imaging device (US UVP gel imaging system) was used to scan the blotted membrane. Using GAPDH as the internal reference, gray-scale analysis was performed.

MSP measurement of DNA methylation of WNT6 gene in cells. MG63 and Hfob1.19 cells were cultured, respectively. 

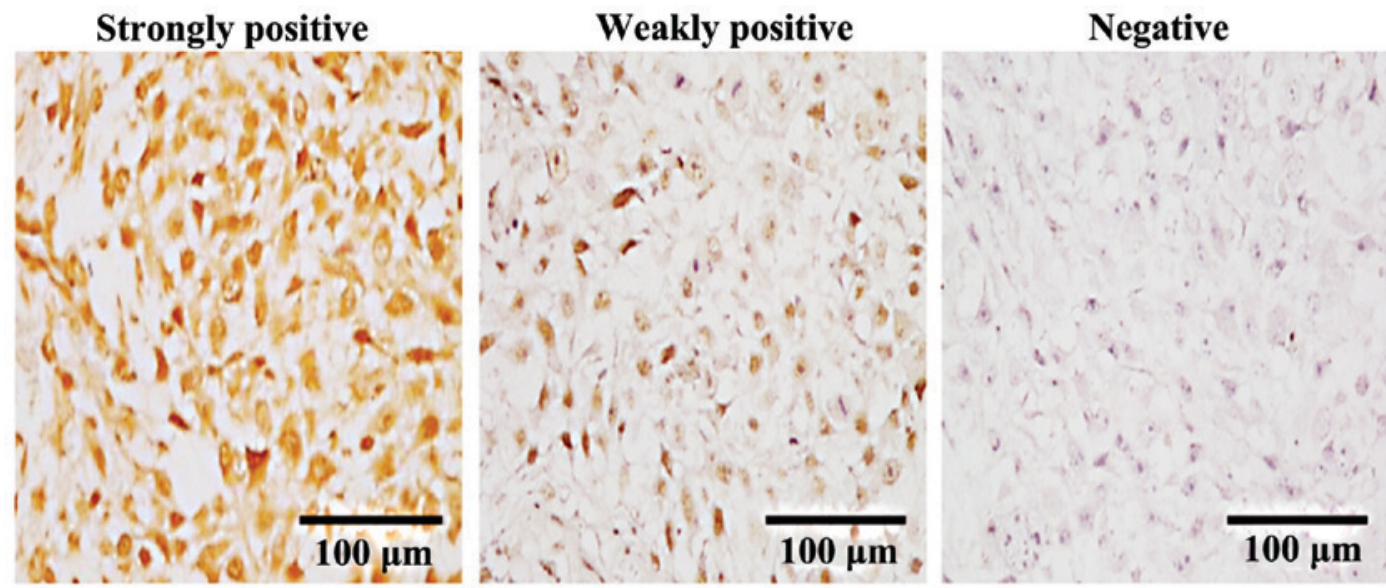

Figure 1. Immunohistochemical assessment of WNT6 protein expression in pathological tissues (magnification, x200).

Table II. WNT6 expression in osteosarcoma tissues.

\begin{tabular}{lllll} 
& \multicolumn{3}{c}{ WNT6 expression } & \\
\cline { 2 - 4 } Cases & - & + & ++ & Positive percentage \\
\hline 50 & 8 & 12 & 30 & $84 \%$ \\
\hline
\end{tabular}

In the exponential phase the cells were digested by trypsin. The total DNA was extracted using the DNA extraction kit from the collected cells. The extracted DNA concentration and purity were determined by UV spectrophotometry. The DNA methylation of WNT6 gene in the cells was determined according to the method in section 2.4.

Correlation of DNA methylation of WNT6 gene and prognosis of osteosarcoma in children. The patients with primary osteosarcoma in children were divided into the WNT6 gene DNA methylation group and WNT6 gene DNA unmethylation group. All the patients were followed up from the time of clinical diagnosis to the time of death or the last follow-up. The effect of DNA methylation of WNT6 gene on prognosis of osteosarcoma in children was analyzed based on the follow-up data.

Statistical analysis. SPSS 17.0 statistical analysis software (IBM Corp., Armonk, NY, USA) was used to process the data. Measurement data were expressed as mean \pm standard deviation and underwent ANOVA single factor variance analysis. Kaplan-Meier analysis of the 5-year survival rate of patients with osteosarcoma was performed based on the clinical prognosis data. Survival curves of patients with osteosarcoma were compared using the log-rank test. The difference was statistically significant at $\mathrm{P} \leq 0.05$.

\section{Results}

Expression of WNT6 protein in primary osteosarcoma in children. The expression of WNT6 protein in primary osteosarcoma could be positive or negative as shown in Fig. 1. Statistical results in Table II showed that the percentage of
Table III. DNA methylation of WNT6 gene in primary osteosarcoma.

Status of DNA methylation of WNT6 gene

\begin{tabular}{lcc}
\cline { 2 - 3 } Cases & Methylated & Unmethylated \\
\hline 50 & $9(18 \%)$ & $41(82 \%)$
\end{tabular}

positive expression of WNT6 protein in primary osteosarcoma was $84 \%(42 / 50)$.

DNA methylation of WNT6 gene in primary osteosarcoma in children. The results in Table III show that the percentage of DNA methylation of WNT6 gene in primary osteosarcoma was $18 \%(9 / 50)$, whereas the percentage of unmethylation was $82 \%(41 / 50)$.

WNT6 protein expression in MG63 and Hfob1.19 cells. Western blot analysis results in Fig. 2 show that WNT6 protein expression in MG63 cells was significantly higher than that in Hfob1.19 cells, and the difference was significant $(\mathrm{P}<0.01)$.

Status of DNA methylation of WNT6 gene in MG63 and Hfob1.19 cells. Results of DNA methylation of WNT6 gene in Fig. 3 showed that the DNA methylation rate of WNT6 gene in MG63 cells was significantly lower than that in Hfob1.19 cells, and the difference was statistically significant $(\mathrm{P}<0.01)$.

Correlation of DNA methylation of WNT6 gene with prognosis of osteosarcoma in children. All the patients were followed up for this study. Among the 50 patients with osteosarcoma in children the 5-year survival rate was 52\% (26/50), of which the 5-year survival rate of patients with DNA methylation of WNT6 gene was 78\% (7/9) and the 5-year survival rate of patients with DNA unmethylation of WNT6 gene was 46\% (19/41). The difference was statistically significant $(\mathrm{P}<0.05)$. From the survival curves, it is evident that the WNT6 gene DNA methylation group had a better prognosis compared with the WNT6 gene DNA unmethylation group (Fig. 4). 
A

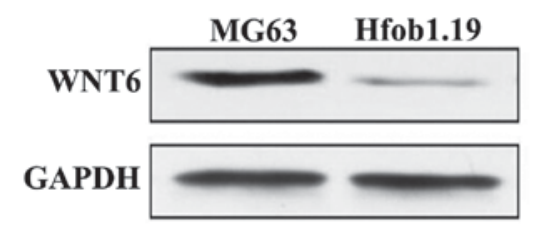

B

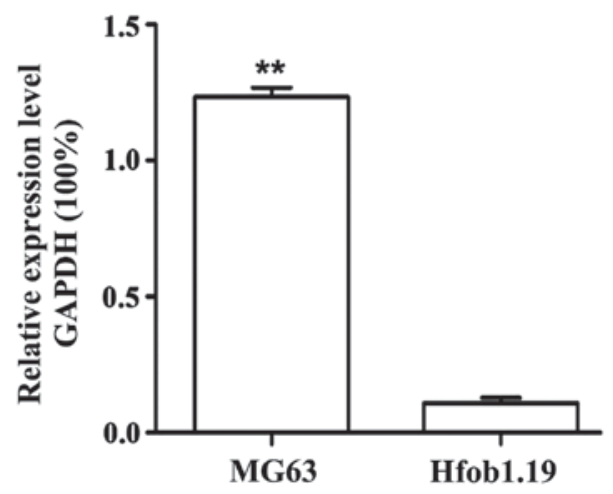

Figure 2. Western blot analysis measurement of WNT6 protein expression in MG63 and Hfob1.19 cells: (A) western blot analysis of WNT6 protein, and (B) gray-scale analysis of western blot analysis results. ${ }^{* *} \mathrm{P}<0.01$.

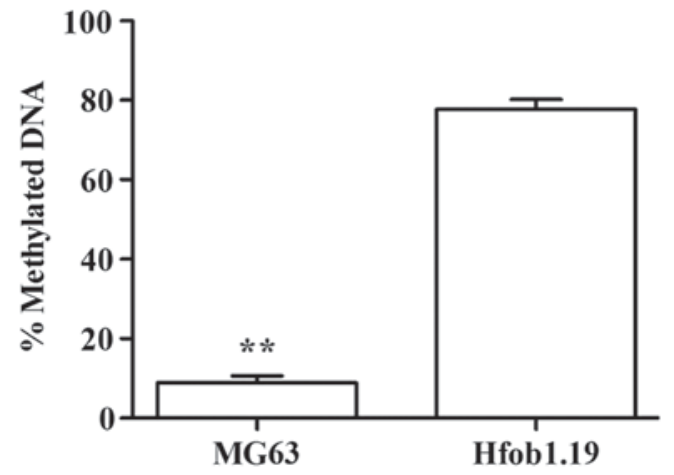

Figure 3. MSP measurement of DNA methylation levels of the WNT6 gene in MG63 and Hfob1.19 cells. MSP, methylation-specific PCR. ${ }^{* *} \mathrm{P}<0.01$.

\section{Discussion}

The occurrence and progress of primary osteosarcoma is a complex process caused by a variety of reasons, including the more recognized factors such as oncogene activation, tumor suppressor gene inactivation and mutation (18). In the study of pathogenesis of osteosarcoma, research was focused on related cell signaling transduction pathways, of which the WNT signaling pathway was one of the most investigated cellular signal pathways. This pathway, not only plays a key role in osteosarcoma, but is also involved in the occurrence and progression of a variety of tumors and is of great significance to clinical prognosis. The WNT6 gene is closely associated with the occurrence and progression of many types of tumors. The WNT6 protein is significantly increased in colorectal, breast and gastric cancer. It is involved in the occurrence and progression and miscorrelated with the level of tumor malignancy (19-21).

When certain genetic mutations occur, tumor formation can be promoted. Epigenetics can affect tumor formation as well. Epigenetics can selectively activate or inhibit the expression of certain genes, and thus participate in various physiological processes in cells. This effect is not achieved by changing the gene nucleotide sequence, instead, mainly by gene DNA methylation, histone modification and gene imprinting (22).

DNA methylation is a common genetic modification in epigenetics, which occurs in tumorigenesis. DNA methylation

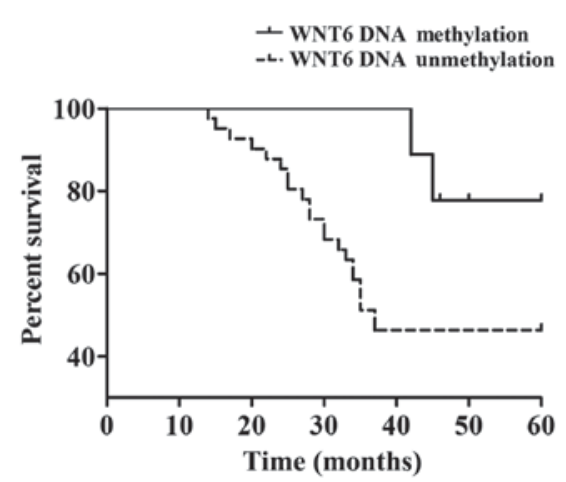

Figure 4. Correlation of DNA methylation of the WNT6 gene with prognosis of osteosarcoma in children.

of suppressor gene can inhibit the transcription process of the gene, and thus affect tumor cell differentiation and proliferation. Research found that abnormal DNA methylation occurs in a variety of tumors $(23,24)$.

In this study, the expression of WNT6 protein in primary osteosarcoma in children was measured by immunohistochemical assay, which showed that $84 \%$ of primary osteosarcoma in children had a positive expression of WNT6 protein. In addition, the DNA methylation status of WNT6 gene in primary osteosarcoma was evaluated by MSP, which demonstrated that the percentages of DNA methylation and unmethylation in primary osteosarcoma were 18 and $82 \%$, respectively. In order to further validate the results, human MG63 osteosarcoma cells and human normal Hfob1.19 osteoblasts were employed in this study. The WNT6 protein expression and DNA methylation of WNT6 gene in the two cell lines were checked by western blot analysis and MSP. It was found that the expression level of WNT6 protein in human osteosarcoma MG63 cells was significantly higher than that in normal human osteoblasts Hfob1.19 $(\mathrm{P}<0.01)$, but the DNA methylation level of WNT6 gene in human osteosarcoma MG63 cells was significantly lower than that in normal human osteoblasts Hfob1.19 ( $\mathrm{P}<0.01)$. The high expression of WNT6 protein in osteosarcoma may due to low DNA methylation level of WNT6 gene.

Moreover, to determine the correlation between DNA methylation of WNT6 gene and prognosis of patients with 
osteosarcoma in children, 50 patients with osteosarcoma in children were followed up for 5 years and the results were statistically analyzed. Among the 50 patients, 9 cases demonstrated DNA methylation of WNT6 gene, of which 2 cases died, and the survival rate was $78 \%$. Forty-one cases demonstrated DNA unmethylation of WNT6 gene, of which 22 cases died, and the survival rate was $46 \%$. Statistical analysis revealed that the DNA methylation level of WNT6 gene has a significant impact on prognosis of patients. A lower DNA methylation level of WNT6 gene was negatively correlated with prognosis of patients. In conclusion, DNA methylation of WNT6 gene may be used as a new biomarker for diagnosis and the prognosis prediction of patients with osteosarcoma in children.

\section{References}

1. Ta HT, Dass CR, Choong PF and Dunstan DE: Osteosarcoma treatment: State of the art. Cancer Metastasis Rev 28: 247-263, 2009.

2. McQueen P, Ghaffar S, Guo Y, Rubin EM, Zi X and Hoang BH: The WNT signaling pathway: Implications for therapy in osteosarcoma. Expert Rev Anticancer Ther 11: 1223-1232, 2012.

3. Longhi A, Errani C, De Paolis M, Mercuri M and Bacci G: Primary bone osteosarcoma in the pediatric age: State of the art Cancer Treat Rev 32: 423-436, 2006.

4. Hayden JB and Hoang BH: Osteosarcoma: Basic science and clinical implications. Orthop Clin North Am 37: 1-7, 2006.

5. Dass CR, Ek ET, Contreras KG and Choong PF: A novel orthotopic murine model provides insights into cellular and molecular characteristics contributing to human osteosarcoma. Clin Exp Metastasis 23: 367-380, 2006.

6. Link MP, Goorin AM, Miser AW, Green AA, Pratt CB, Belasco JB, Pritchard J, Malpas JS, Baker AR, Kirkpatrick JA, et al: The effect of adjuvant chemotherapy on relapse-free survival in patients with osteosarcoma of the extremity. N Engl J Med 314: 1600-1606, 1986

7. Paul S and Dey A: Wnt signaling and cancer development: Therapeutic implication. Neoplasma 55: 165-176, 2008.

8. Cleton-Jansen AM, Anninga JK, Briaire-de Bruijn IH, Romeo S, Oosting J, Egeler RM, Gelderblom H, Taminiau AH and Hogendoorn PC: Profiling of high-grade central osteosarcoma and its putative progenitor cells identifies tumourigenic pathways. Br J Cancer 101: 1909-1918, 2009.

9. Kirikoshi H, Sekihara $\mathrm{H}$ and Katoh M: WNT10A and WNT6, clustered in human chromosome 2 q35 region with head-to-tail manner, are strongly coexpressed in SW480 cells. Biochem Biophys Res Commun 283: 798-805, 2001.

10. Katoh M: WNT and FGF gene clusters (Review). Int J Oncol 21 1269-1273, 2002.
11. Lavery DL, Davenport IR, Turnbull YD, Wheeler GN and Hoppler S: Wnt6 expression in epidermis and epithelial tissues during Xenopus organogenesis. Dev Dyn 237: 768-779, 2008.

12. Geetha-Loganathan P, Nimmagadda S, Huang R, Scaal M and Christ B: Role of Wnt-6 in limb myogenesis. Anat Embryol (Berl) 211: 183-188, 2006.

13. Wang C, Ren L, Peng L, Xu P, Dong G and Ye L: Effect of Wnt6 on human dental papilla cells in vitro. J Endod 36: 238-243, 2010.

14. Lavery DL, Martin J, Turnbull YD and Hoppler S: Wnt6 signaling regulates heart muscle development during organogenesis. Dev Biol 323: 177-188, 2008

15. Nakatsu MN, Ding Z, Ng MY, Truong TT, Yu F and Deng SX: Wnt $/ \beta$-catenin signaling regulates proliferation of human cornea epithelial stem/progenitor cells. Invest Ophthalmol Vis Sci 52: 4734-4741, 2011.

16. Hao J, Li TG, Qi X, Zhao DF and Zhao GQ: WNT/ $\beta$-catenin pathway up-regulates Stat 3 and converges on LIF to prevent differentiation of mouse embryonic stem cells. Dev Biol 290: 81-91, 2006.

17. Galbraith RL, Poole EM, Duggan D, Muehling J, Hsu L, Makar K, Xiao L, Potter JD and Ulrich CM: Polymorphisms in WNT6 and WNT10A and colorectal adenoma risk. Nutr Cancer 63: 558-564, 2011.

18. Messerschmitt PJ, Garcia RM, Abdul-Karim FW, Greenfield EM and Getty PJ: Osteosarcoma. J Am Acad Orthop Surg 17: $515-527,2009$.

19. Krawetz R and Kelly GM: Wnt6 induces the specification and epithelialization of F9 embryonal carcinoma cells to primitive endoderm. Cell Signal 20: 506-517, 2008.

20. Yuan G, Regel I, Lian F, Friedrich T, Hitkova I, Hofheinz RD, Ströbel P, Langer R, Keller G, Röcken C, et al: WNT6 is a novel target gene of caveolin-1 promoting chemoresistance to epirubicin in human gastric cancer cells. Oncogene 32: 375-387, 2013.

21. Qurrat-U1-Ain, Seemab U, Nawaz S and Rashid S: Integrative analyses of conserved WNT clusters and their co-operative behaviour in human breast cancer. Bioinformation 7: 339-346, 2011.

22. Kohno H, Amatya VJ, Takeshima Y, Kushitani K, Hattori N, Kohno N and Inai K: Aberrant promoter methylation of WIF-1 and SFRPI, 2, 4 genes in mesothelioma. Oncol Rep 24: 423-431, 2010.

23. Guo Y, Guo W, Chen Z, Kuang G, Yang Z and Dong Z: Hypermethylation and aberrant expression of Wnt-antagonist family genes in gastric cardia adenocarcinoma. Neoplasma 58: 110-117, 2011.

24. Wu D, Wong P, Li W, Vogel CF and Matsumura F: Suppression of WIF-1 through promoter hypermethylation causes accelerated proliferation of the aryl hydrocarbon receptor (AHR) overexpressing MCF10AT1 breast cancer cells. Toxicology 285: 97-103, 2011. 